\title{
Magnetic Reconnection of Plasma Toroids with Co- and Counter-Helicity
}

\author{
M. Yamada," Y. Ono, A. Hayakawa, M. Katsurai \\ Department of Electrical Engineering PPL- 2688 \\ University of Tokyo \\ Tokyo, Japan \\ DE90 013501
}

and

F. W. Perkins*

Princeton Plasma Physics Laboratory

Princeton, New Jersey 08544

\begin{abstract}
Magneic reconnection phenomena are investigated raking into account all three vector components of the magnetic field in a laboratory experiment. Two toroidal magnetized plasmas carrying identical toroidal currents and poloidal field configurations are made to collide, thereby inducing magnetic reconnection. The directions of the toroidal field play an important role in the merging process. It is found that plasmas of anti-paraliel helicity merge much faster than those of parallel helicity. It is also found that the reconnection rate is proportional to the initial relative velocity of the two plasma tori, suggesting that magnetic reconnection, in the present experiment, is a forced pheoromenon.
\end{abstract}

\section{DISCLAIMER}

This repart was prepared as an account of work sponsored by an agency of the Uniled States This repon was prepared as an acoout of work spenter nor any agency thereof, nor any of their

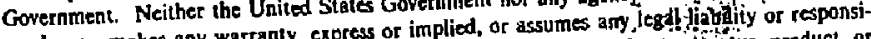
employees, makes any wastanty, express or implied, or ass information, 'eppitatus, product, or bility for the eccuracy, completencss, ur usefuiness of any infort process "sclosed, or represents that its use would not infrice privalely orade name, trademark, ence herein to sny specific commercial product, process, or seritu by its endorsement, recommanufacturer, or otherwise does not necessarily consthe or inply inency thereof. The views mendation, or favoring by the United States Government of any agency therest The of the and opinions of authors expressod herein do not 
Magnetic field line reconnection plays an important role in many plasma physics phenomena in the universe, ${ }^{1}$ such as the evolution of solar flares, ${ }^{2}$ development of the earth's magnetosphere ${ }^{3}$ and magnetic relaxation in laboratory plasmas for nuclear fusion research. ${ }^{4}$ To elucidate the complicated evolution of the magnetic field lines in a simple way, magnetic reconnection, in early research in astrophysics and solar physics, was ofen analyzed as a twodimensional local phenomenon. In laboratory plasmas, such as in devices for magnetic fusion research, 4,5 it has often been investigated as a global phenomenon -- monitoring the total magnetic flux, helicity, and energy of the magnetically confined plasmas. Its local features have not been seen due to the difficulty in direct measurement of the internal structure of the magnetic field lines.

The present paper addresses two important issues: a) how the third-dimensional component of the magnetic field line affects the reconnection, and b) how the global plasma characteristics influence the local fearures of the reconnection. Pertinent to the results of the present experiment is a recent computer simulation ${ }^{6}$ that examined the reconnection of field lines merging with many different angles.

The most commonly used description of magnetic field line reconnection is shown in Fig. 1(a), based on two-dimensional analyses of magnetic fieid evolution as made by Sweet, Parker, and Petschek. 7,8 In actual reconnection phenomena, the magnetic field lines have significant components in all three dimensions, as observed in solar flares and in most laboratory experiments. For example, the same 2D picture of the field line shown in Fig. 1(a), describing the merging of two plasma toroids carrying equal currents, appears quite differently in the 3D sketches shown in Figs. 1(b) and 1(c). Even though their 2D representations are identical, the three-dimensional pictures of the merging of two otherwise identical toroidal plasmas differ strongly, depending on whether their initial helicities were parallel or anti-parallel. In the former case, the field lines merge at various angles, while in the latter case the field lines merge exactly with anti-parallel symmetry. In addition, the internal toroidal field is necessarily accompanied by a poloidal plasma current and the additional $\mathbf{j} \times \mathbf{B}$ force changes the character of the magnetic reconnection. In general, in the case of merging counter helicities, there is a parallel poloidal current on both sides of the reconnection region, while the current flows with an angle to each other for co-helicity merging.

There is another important difference in the reconnection patrems shown in Figs. 1(b) and Fig. 1(c). Conserving helicity, the transition from the configuration of Fig. l(b) to Fig. 1(b') 
should be globally smooth. In the case of counter-helicity merging, Fig. l(c) and Fig. l(c'), the pitch of the field lines changes abruptly at the reconnection point. One expects violent plasma acceleration in the toroidal direction as the field lines contract after reconnectioti (a slingshot effect).

Another outstanding issue is to determine how the reconnection rate is related to the local and global plasma parameters. Sophisticated experiments will be necessary to determine these connections. Sorrie preliminary but important findings are reported in this paper.

Recently a comprehensive experiment has been proposed to investigate effects of threedimensional magnetic field line reconnection on the Proto-S1 spheromak device. ${ }^{9}$ To identify critical issues, preliminary experiments have been carried out in the TS-3 spheromak device at the University of Tokyo. 10 A related study had already been carried out on this device, investigating the global characteristics of merging spheromaks. 11 Figure 2 shows the set-up for the present experiment in which two spheromak plasmas of toroidal shape are created and allowed to merge together. In the vacuum vessel there are eight sets of electrode pairs and a poloidal field coil of $22 \mathrm{~cm}$ radius. The toroidal flux in the each spheromak is generated by the $z$-discharge current between the electrodes, while the poloidal fluxes are induced by the poloidal field coil currents. The formation of this " $z-\theta$ " pinch type spheromak 12 is completed in $30 \mu \mathrm{sec}$, after which the plasma current is sustained for $70 \mu \mathrm{sec}$ with a help of Ohmic heating induction by a cerivial solenoid. The two spheromaks can have magnetic helicities ${ }^{5}$ of

$$
\mathrm{K}= \pm c \psi_{\mathrm{S}} \phi_{\mathrm{S}}
$$

in which $\psi_{S}$ and $\phi_{S}$ are the poloidal and toroidal fluxes contained in the spheromak plasmas, and $c$ is a profile factor. The polarity of $\mathrm{K}$ for the two spheromaks is determined independently by the direction of the $\mathrm{z}$-discharge currents of the toroidal fields. The average plasma density is about $3 \times 10^{14} \mathrm{~cm}^{-3}$ ( for hydrogen and helium discharges), the electron temperature $T_{e}=5 \sim 15 \mathrm{eV}$, the peak toroidal field $\mathrm{B}_{\mathrm{t}} \leq 1 \mathrm{kG}$, the average $\angle B>\leq 20 \%$, the magnetic Reynolds number $S=500$, and the coroidal plasma current $I_{p t} \approx 30-50 \mathrm{kA}$. To investigate magnetic field line reconnection in the neighborhood of the midplane, $z=0$, the plasmas of $R_{p} \sim 15 \mathrm{~cm}$ and $r_{p} \sim 8-10 \mathrm{~cm}$ are made to collide. Ion gyro-radii are much smaller $(2-5 \mathrm{~mm})$ than the plasma sizes. To document the internal magnetic structure of the reconnection on a single shot, a two-dimensional magnetic probe array is placed on an $r-z$ plane of the vessel. This $5 \times 7$ array (grid spacing $5 \mathrm{~cm} \times 5 \mathrm{~cm}$ ) is composed of 35 small pick-up coils inside five glas: tubes of 
$5 \mathrm{~mm}$ diameter. Signals from additional monitoring probes showed this array did not disturb the plasma magnetics by large amount $(\delta \mathrm{B} / \mathrm{B} \leq 5 \%)$. In a second set-up, two spheromaks generated by co-axial guns collided together in a similar way as the earlier experiments had been performed to study global MHD phenomena. ${ }^{13}$ Approximately the same results were obtained although the data were less reproducible.

Initiating three-dimensional analysis of magnetic reconnection in a laboratory experiment, the present study focussed on (i) helicity questions, that is, the effects of the third (oroidal) component of the magnetic field and (ii) the effect, on the reconnection rate, of the relative velocities of the merging plasmas.

In Fig. 3, the merging of two toroidal plasmas of the same helicity $(K+K)$ is compared with the merging of opposite helicities $[K+(-K)]$. The figure shows the time evolution of the poloidal flux contours derived experimentally from intemal probe signals for the merging of coand counter-helicities. Other plasma parameters were held identical for each discharge. A merging of spheromaks of opposite helicity is shown to be more efficient compared to merging of the same helicity. In agreement with the expectation mentioned above, opposite helicities are seen to merge rapidly and sometimes violently. The merging is often accompanied by a sinusoidal oscillation of $100 \mathrm{kHz}$ whose dominant toroidal mode number was measured to be $\mathbf{n}=1$ and/or $\mathbf{n}=2$. The phase velocity of the mode is $1-2 \times 10^{7} \mathrm{~cm} / \mathrm{sec}$, roughly equal to valfven. Merging of two spheromaks with the same helicity occurs rather smoothly and the total helicity of the spheromaks is approximately conserved, which was observed in the earlier experiment. "]

In the case of co-helicity merging, the reconnection rate is seen to slow down significantly after $\mathrm{t}=40 \mu \mathrm{s}$, while for counter-helicity merging, reconnection continues until they merge complerely, Fig. 3. During the initial phase, reconnection progresses with the same speed for both.

To describe the reconnection process quanrisatively, we define $\psi_{c}$ and $\psi_{p}$ as ihe values of the highest common flux and peak flux of the each plasma. We then define the cornmon flux ratio $\alpha_{c}$

$$
\alpha_{c}=\Psi_{c} / \Psi_{p}
$$

If the peak flux values of two plasmas do not agree (generally $\Delta \psi_{p} / \psi_{p}<0.1$ ), the smaller value is used. A complete merging refers to $\alpha_{c}=1.0$. Monitoring $\alpha_{c}$ versus time, one can then 
quantify the rate of magnetic field reconnection by $\mathrm{d} \alpha_{C} / \mathrm{dt}$. In the present study, reconnection is analyzed as a local phenomenon between the two plasmas and we count $\psi$ only inside the separatrix region shown in Fig. 3. Figure 4 depicts $\alpha_{c}$ versus time for various colliding velocities for counter-helicity merging.

It is generally observed that $\alpha_{C}$ increases initially with almost the same speed for co- and counter-helicity merging, but the reconnection rate slows down significantly after $\alpha_{C}$ reaches $50 \%$ in co-helicity merging, while it progresses with approximately the same speed in counterhelicity merging until it reaches $100 \%$ as seen in Fig. 3 . Here one should note that the angle of the merging field lines changes gradually from $180^{\circ}$ to $0^{\circ}$ for co-helicity merging as reconnecion progresses, because the rotational transform of the flux hole spheromak varies radially $(q=0$ at the edge and $q=0.6$ at the magnetic axis, ${ }^{9}$ where $q$ is the inverse of the rotational cransform). For counter-helicity the angle is always $180^{\circ}$. The recent computer simulation ${ }^{6}$ concluded that the reconnection occurred most efficiantly for a merging angle of $180^{\circ}$ and least for $0^{\circ}$, with which the observed inefficiency of co-helicity merging in the later phase is consistent .

Another significant result of the present experiment is the observation of a strong dependence of the reconnection rate on the relative speed of approach of the two plasmas, as seen in Fig. 4. The speed, which is much smaller than valfven, can be controlled by adjusting the poloida: bias field or by the ejection speed of gun plasmas and is an important parameter in recognizing forced reconnection. In the present set-up the force is estimated to be approximately proportional to the merging velocity, $v_{m}$, based on our earlier spheromak formation experiments; $9.10\left[v_{m} \sim-I_{P F} \sim B_{T}(e x t), F_{z}=I_{p} \times B_{T}(e x t)\right.$, thus $F_{z} \sim v_{m}$ for constant plasma current $I_{p}$ ]. Figure 5 presents the reconnection rate of two plasmas versus initial relative speed $v_{m}$ for co- and counter-heliciry merging. The reconnection rate is defined as time derivative of $\mathrm{d} \alpha_{\mathrm{c}} / \mathrm{dt}=\gamma_{\mathrm{R}}$ between $\alpha_{\mathrm{C}}=40 \%$ and $80 \%$. As seen Fig. 5, $\gamma_{\mathrm{R}}$ increases proportionally with $v_{\mathrm{m}}$. The dependence is seen in three independent experiments; a $z-\theta$ discharge merging with supplemental current drive, without current drive and in a two-gun merging experiment. This trend clearly suggests the importance of an external driving force and supports an important aspect of a driven reconnection model. 6 In recent tokamak experiments, a very fast magnetic reconnection ( $\tau_{\mathrm{rec}}<50$ fisec) has been cbserved during internal disruptions, and the piesent results might support the notion that fast plasma flow near the $q=1$ surface induces the fast reconnection. 14 
In conclusion, three important physical features of magnetic reconnection can be extracted from the experimentally derived flux contour plots. (i) The third-dimensional component of the magnetic field plays an important role in the magnetic reconnection, (ii) counter-helicity merging induces magnetic reconnection more effectively than co-helicity merging, and (iii) the reconnection rate is proportional to the initial approaching speed of two toroidal plasmas. Further study to determine the dependence on local ${ }^{15}$ and global ${ }^{16}$ stucture is now needed to give a full picture of magnetic reconnection in three dimensions.

\section{Acknowledgment}

The authors would like to thank Drs. W. Park, T. Stix, and S. Yoshikawa for fruitful discussions and especially Tom Stix for critical reading of this manuscript. Experiments were carried out during the visit of one of the authors (M. Y.) at the University of Tokyo on Fellowship from Japan Society for Promotion of Scientific (JSPS).

This work is partly supported by the U.S. Department of Energy Contract No. ACO2-76-CHO3073. 


\section{References}

1V. M. Vasyliunas, Rev, Geophys. Space Phys. 13, 303-336 (1975).

2E. N. Parker, Astrophysical J. 180, 247 (1973); E. R. Priest, "Solar Magnetohydrodynamics," (P. Reidel, Dordrecht, 1984), Chap. 10.

3Y. Shi et.al., Geophys. Rev. Lett. 15, 195 (1988).

4J. A. Wesson, "Tokamaks" (Clarendon Press, Oxford, 1987), p. 176-183.

5J. M. Finn and T. Antonsen, Comments on Plasma Phys. and Contr. Fus. 2, 111 (1985);

J. M. Finn, Phys. Fluid 29, 2630 (1986).

6T. Sato, Phys. Rev. Lett. 54, 1502 (1985).

7P. A. Sweet, Nuouo Cim. Suppl. 8, 188 (1958); E. N. Parker, J. Geophys, Rev. 62, 509 (1957).

${ }^{8}$ H. E. Petschek, "Magnetic Field Annihilation," NASA Spec. Pub. SP-50, 425 (1964).

9M. Yamada, et al., Bull. Am. Phys. Soc. 34, 2061 (1989); M. Yamada et al., Phys. Rev.

Lett. 46, 188 (1981).

10Y. Ono, et al., IEEE Trans. Plasma Science, Vol. PS-15, 418 (1987).

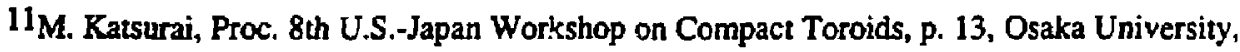
Japan (1986).

12G. Goldenbaum et al., Phys. Rev. Lett. 44, 393 (1980).

13K. Kawai, University of Washington, docioral thesis (1988); E. E. Nolting et al., J. Plasma Phys. 2.1 (1973).

${ }^{14}$ K. McGuire, et al., Proc. 11th Int. Conf. on Plasma Physics and Controlled Nuclear Fusion Research, Japan (1986), Vol. 1, 421, IAEA, Vienna, 1987.

15 R. L. Stenzel and W. Gekelman, J. Geophys. Rev. 86, 649 (1981).

16J. B. Taylor, Rev. Mod. Phys. 58, 741 (1986). 
Figure captions

Fig. 1. Three-dimensional effects of magnetic reconnection. Fig. 1(a), 2D local poloidal picture of magnetic field line at the reconnection point; Fig. 1(b) and Fig. 1(b'), 3D description of evolution for merging two toroidal plasmas with equal helicity, before and after reconnection; Fig. 1(c) and Fig. 1(c'), 3D description of evolution for two plasmas with opposite helicity, before and afier recoanection.

Fig.2. Experimental set-up in TS-3 device. The central column provides stability effects for spheromaks with a flux-hole (currentless region) at the major axis.

Fig. 3 Evolution of poloidal flux contours for co- and counter-heliciry merging. The other plasma parameters are kept identical for the cascs shown. The total plasma current, $I_{p}=35-50 \mathrm{kAmp}$.

Fig.4. Common flux ratio $\alpha_{c}$ versus time for reconnection of two counter-helicity plasmas.

Fig. 5. Measured reconnection rate versus mutual colliding velocity $\mathrm{v}_{\mathrm{m}}$ of two plasmas for coand counter- helicity merging. 
$\$ 90 \times 0056$

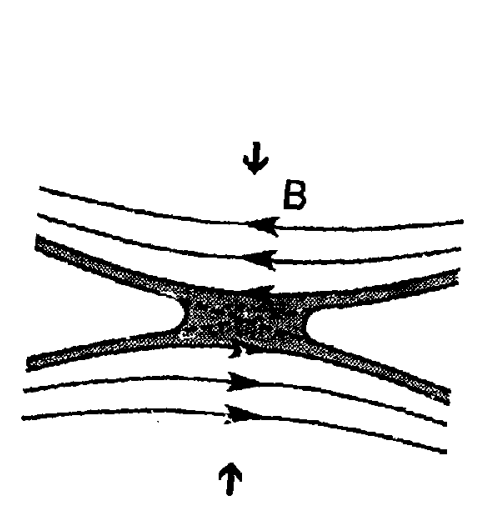

(a)

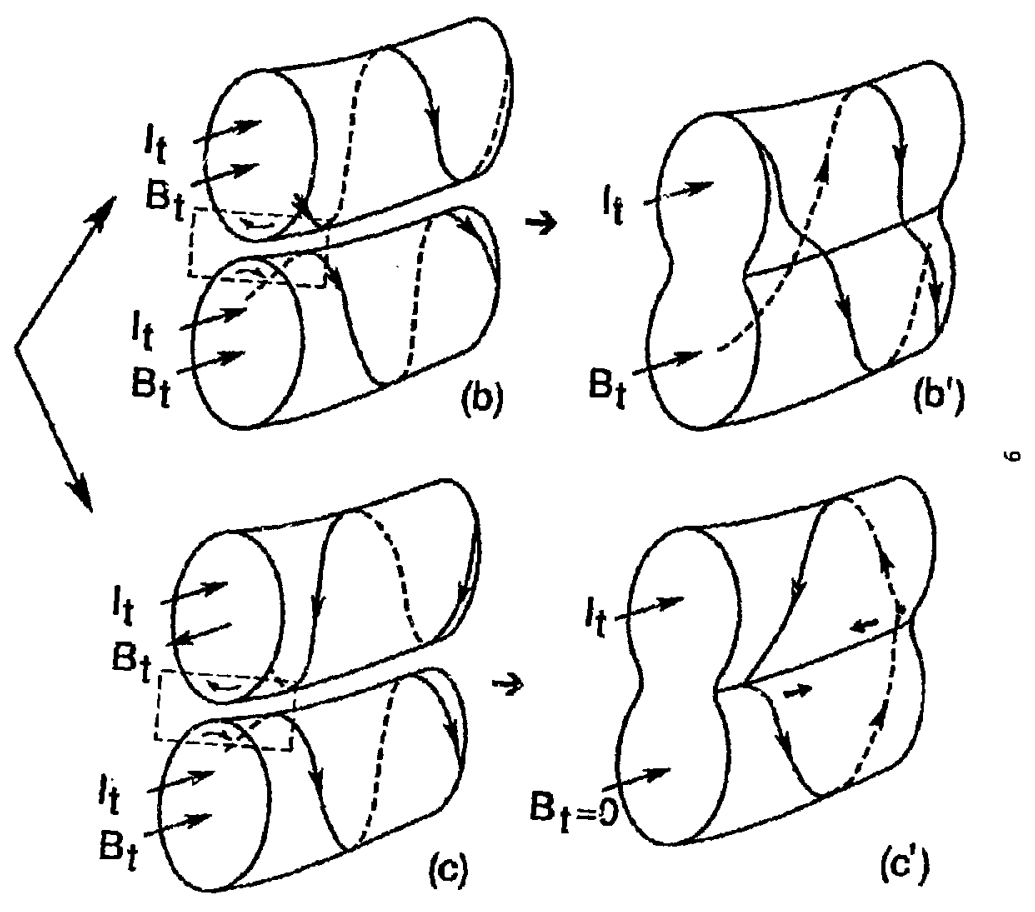

FIG. 1 
\#90X0155

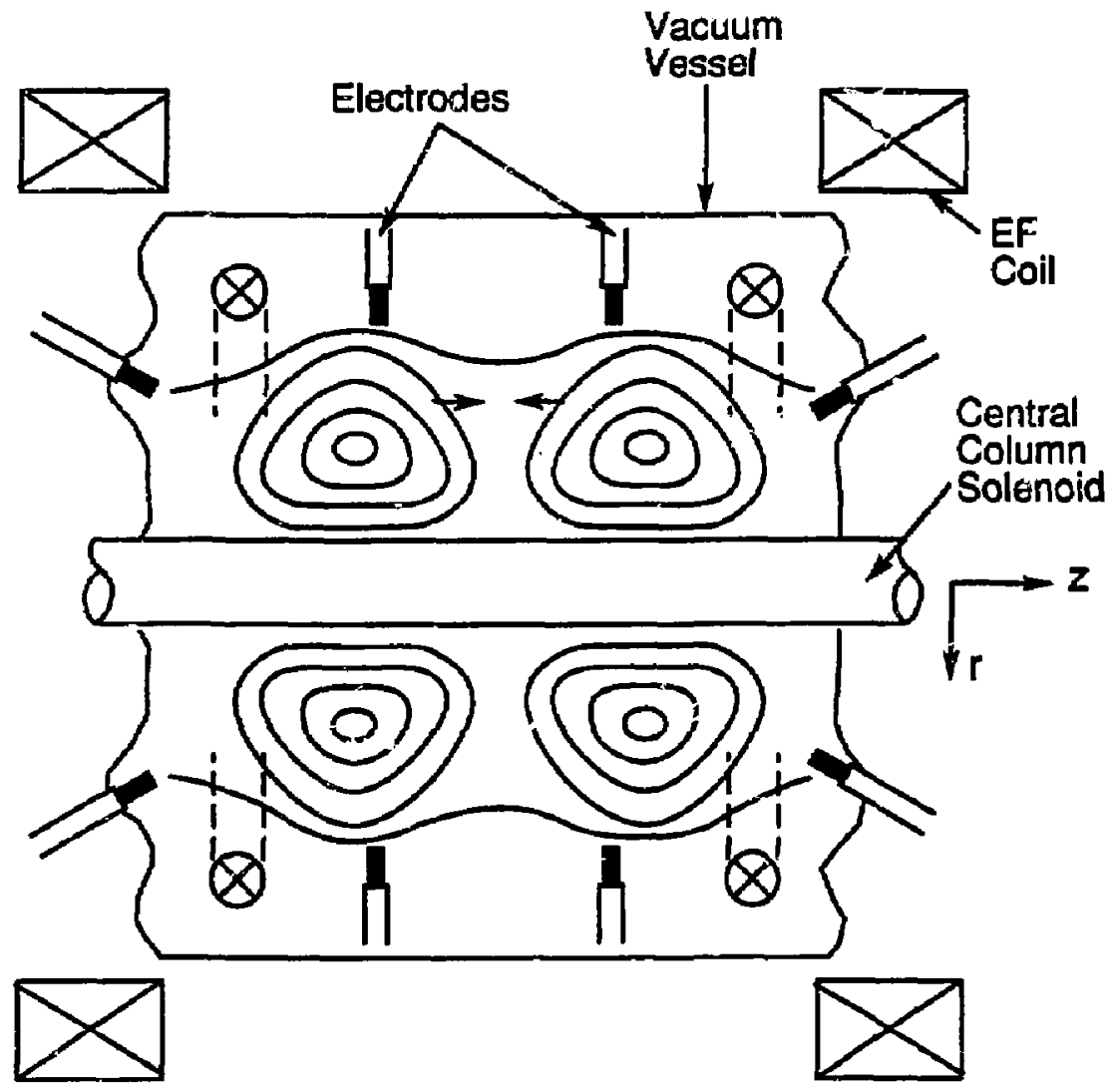



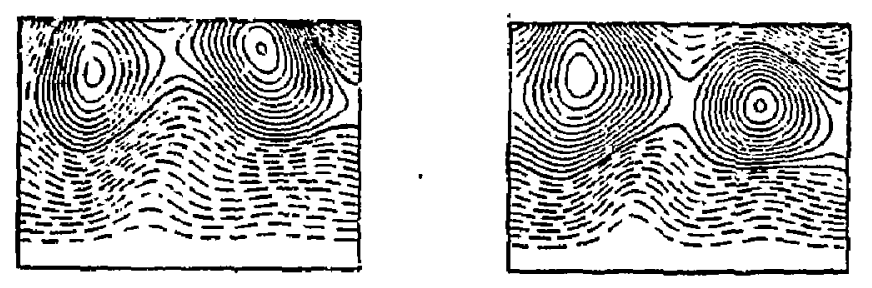

$90 \times 3054$
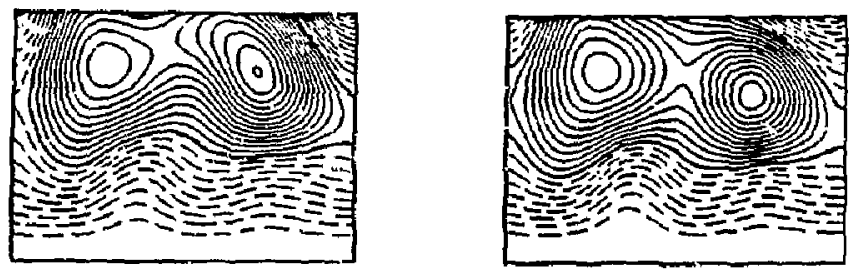

$t=30 \mu \mathrm{sec}$
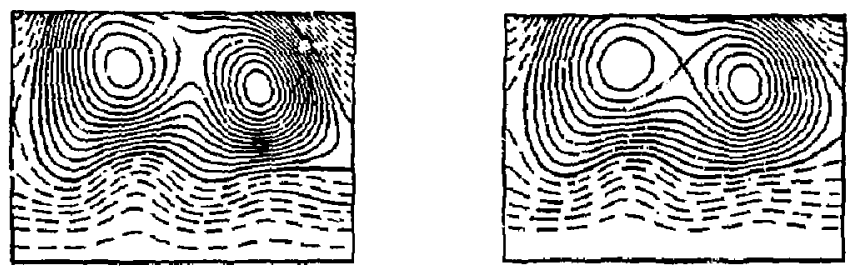

$t=35 \mu \mathrm{sec}$
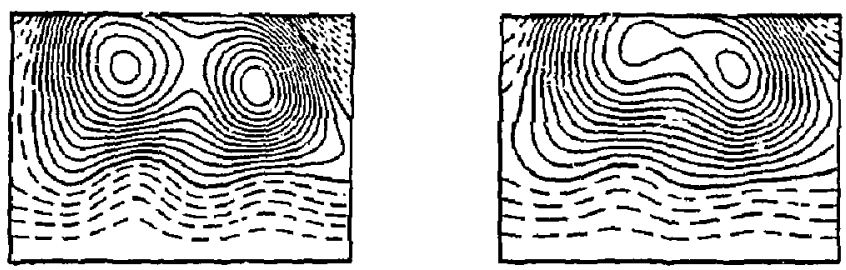

$t=40 \mu \mathrm{sec}$
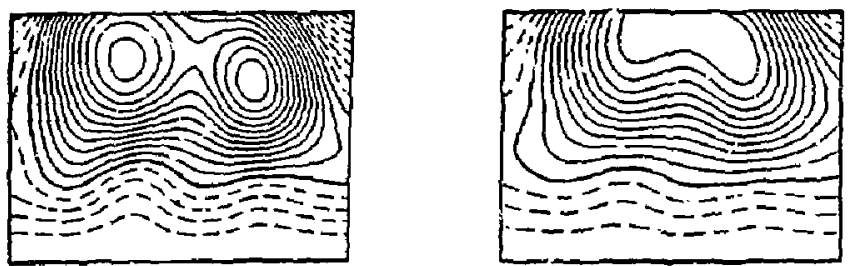

$t=45 \mu s e c$

Co-Helicity Merging

Counter-Helicity Merging 


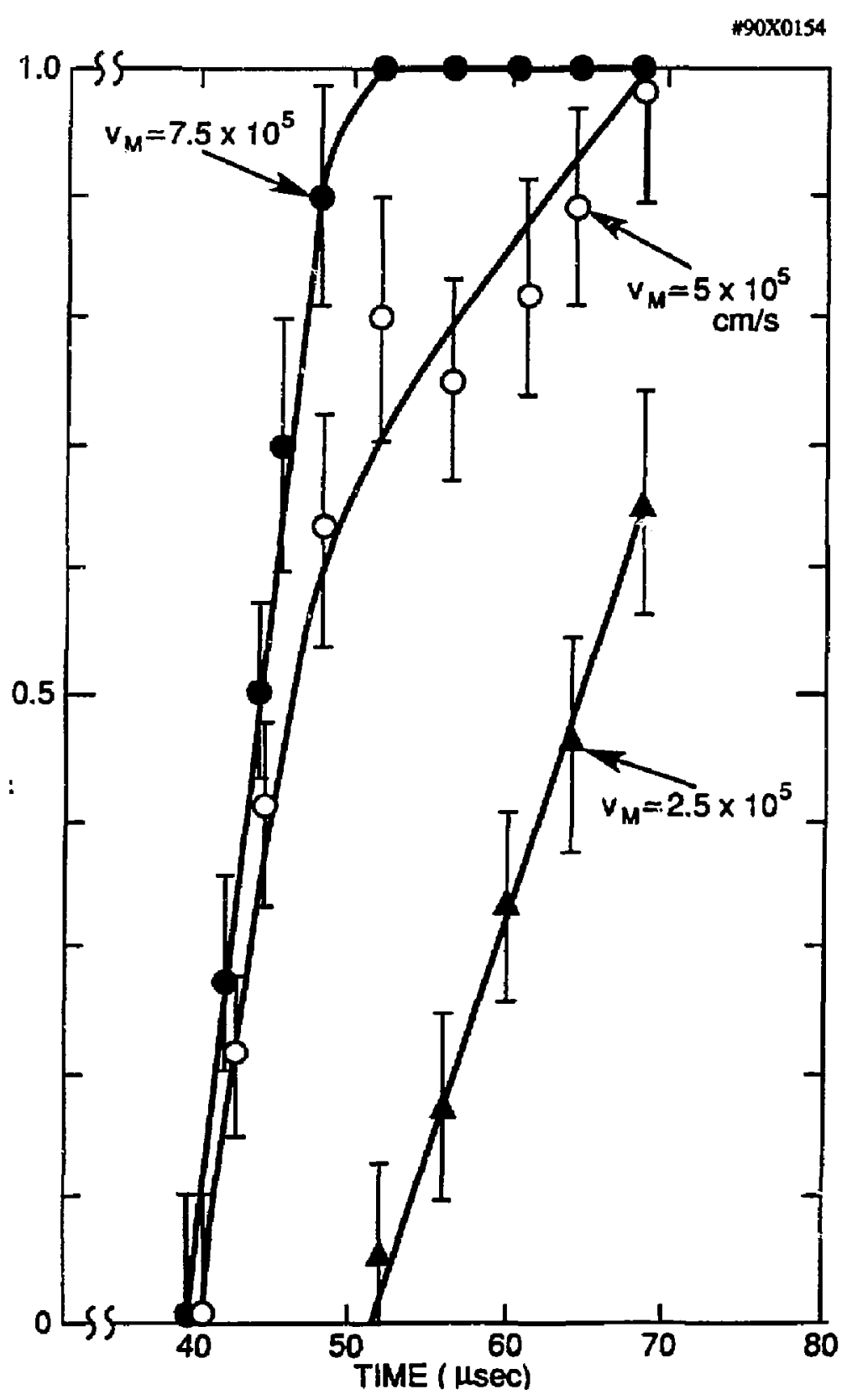

FIG, 4 


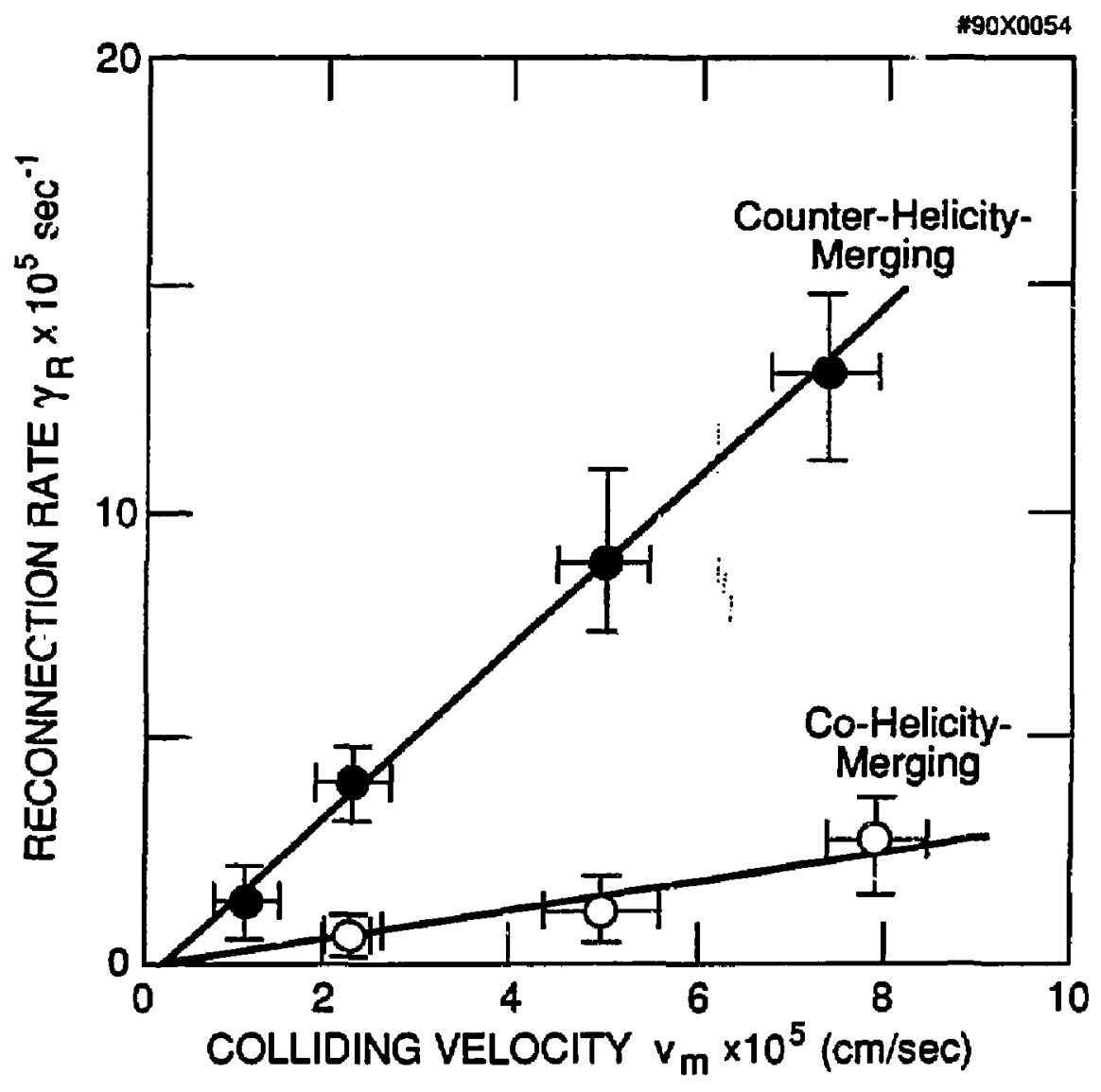

FIG. 5 